Aim of the study: The aim of this study is to research the contribution of radiothermometry (RTM) to the characterization of breast masses, the differentiation of malignant-benign masses and diagnosis of early stage breast cancer.

Material and methods: This prospective study comprised 182 cases of patients diagnosed with a breast mass and a control group of 55 cases: a total of 237.

Results: When histopathology is accepted as the gold standard among diagnostic methods, the sensitivity, specificity, and positive-negative predictive value for RTM were $90.9 \%$, $20.8 \%, 61.2 \%$ and $62.5 \%$, respectively. Consistency was 0.129 . When compared with mammography the same values for RTM examination are $87 \%$, $81.4 \%, 58 \%$ and $95.5 \%$. Consistency was 0.582 . Evaluating with respect to size of the mass accepting mammography as the gold standard, RTM examination had sensitivity, specificity, positive and negative predictive values for masses $2 \mathrm{~cm}$ and above of $88.9 \%, 83.3 \%, 88.9 \%$ and $83.3 \%$. Consistency was 0.722 . The consistency of RTM for lesion diagnosis in BI-RADS II breast structure is higher than the consistency of mammography.

Conclusions: Identification of lesions in the breast and presence of microcalcification by RTM shows that it is more trustworthy compared to mammography. When compared with mammography the validity results for RTM show there is a good level of conformity between the two methods. When evaluated based on the area below the ROC cure and compared to mammography, RTM is sufficiently successful at evaluating positive and negative cases.

Key words: radiothermometer, breast cancer, radiometry, mammography, ultrasonography.

Contemp Oncol (Pozn) 2014; 18 (3): 197-203 DOI: $10.5114 /$ wo.2014.42721

\section{Efficacy and safety of breast radiothermometry in the differential diagnosis of breast lesions}

\author{
Sahnaz Caferova ${ }^{1}$, Fatma Uysal ${ }^{1,2}$, Pınar Balcı $^{1}$, Serdar Saydam ${ }^{3}$, Tülay Canda ${ }^{4}$
}

${ }^{1}$ Department of Radiology, Dokuz Eylül University, Izmir, Turkey

${ }^{2}$ Çanakkale Onsekiz Mart University, Çanakkale, Turkey

${ }^{3}$ Department of General Surgery, Dokuz Eylül University, Izmir, Turkey

${ }^{4}$ Department of Pathoology, Dokuz Eylül University, Izmir, Turkey

\section{Introduction}

Breast cancer, the most common form of cancer in women together with lung cancer, continues to be an important cause of mortality and morbidity. According to the American Cancer Society, breast cancer is the most frequently diagnosed cancer in women in the US and is the second highest cause of cancer-related deaths in women [1, 2].

Mammography, with $90 \%$ sensitivity for screening and identifying breast lesions, is still the most basic method. However, in some cases, lesions that are palpable on clinical examination may remain hidden on mammography due to superposition of breast tissue. Sometimes mammography size was smaller than histopathological size [2]. Additionally, more than $75 \%$ of lesions identified on mammography are diagnosed as pathologically benign. The most important problem is that the sensitivity of mammography to identify lesions is reduced in situations with dense breast tissue due to history of radiotherapy or surgery, breast tissue adjacent to implants and dense breast tissue in the young population. In this situation the first method to investigate the breast is ultrasonography. This method is very successful for lesion identification, especially for differentiating cystic and solid masses; however, the most important limitation is that it cannot identify microcalcification and ductal carcinoma in situ [2-4].

A thermal imaging system, breast radiothermometry mammography (RTM), which measures and analyzes the breast surface (infrared thermometry) and internal temperature (microwave thermometry), is a sensitive method that aids the diagnosis of breast cancer. The RTM devices measure electromagnetic waves sent through the breast tissue to identify the diseased area. The microwave thermometry method is a new technology developed in recent years. This method can diagnose cancer using the thermal activity of cancer cells before they have caused any structural changes in breast tissue. It has the potential to distinguish between fibrocystic cell changes and cancer cell changes. Investigation does not involve radiation, and all areas can be imaged including the area where the breast meets the arm $[5,6]$.

There is no definite preventive method for breast cancer yet. The current aim is early diagnosis. Due to early diagnosis, many of the problems caused by breast cancer can be solved, reducing the damage to the patient population to a minimum and increasing duration and quality of life appreciably.

The aim of this study is to research the contribution of RTM to the characterization of breast masses, the differentiation of malignant-benign masses and diagnosis of early stage breast cancer. 


\section{Material and methods}

This prospective study comprised 182 patients who applied to the Mammography unit of the Radiology Clinic of Dokuz Eylul University Medical Faculty Hospital and were diagnosed with a breast mass and a control group of 55 cases: a total of 237.

According to mammographic and ultrasonography evaluation 57 cases were pre-diagnosed with Breast Imaging-Reporting and Data System (BI-RADS) IV and BI-RADS $\checkmark$ and were biopsied and histopathologic scans were completed. Sixty cases were diagnosed as BI-RADS II lesions and 65 cases were diagnosed as BI-RADS III and monitored.

Mammographic investigation used the "Hologic-Selenia Digital Mammography System" in the craniocaudal (CC) and mediolateral-oblique (MLO) positions. Extra positions for investigation were used for patients requiring them. Breast ultrasonography investigations used a (Philips HD11 USG) Doppler ultrasonography (US) device 5-12 MHz high-resolution linear probe and were completed simultaneously to mammography. After patients signed the informed consent form, radiothermometry examination was completed using a Computer Based Radiometer for Measuring Integral Internal Temperature (RTM-01-RES) device. Investigations were completed by an operator blind to the results of the mammography. Before examination, room temperature was fixed at $23-25^{\circ} \mathrm{C}$. Patients removed clothing above the waist and lay supine with hands under the head for 10 minutes to adjust to the room temperature. Each breast was measured in 9 different regions and in the axilla region. As recommended in the literature [7], to attain body temperature the probe was first placed on the epigastrium. The probe axis was held perpendicular to the skin surface and the full surface was touched to the skin. It was held without pressure for 15 seconds. The start of the probe sensing temperature was shown by a line forming on the screen. Once the warning signal was heard the probe was placed on the other breast. Both breasts were measured at 15-second intervals. Of the total of 237 patients who underwent RTM examination a temperature increase was noted in 128 (55\%) (Fig. 1).

Measured RTM results were shown on the monitor in diagram form. To image the areas of internal heat, the names of measured points were shown on the horizontal axis with the corresponding internal temperatures shown on the vertical axis.

Data were displayed on the monitor with different colors. The cool areas of the breast were shown with cold colors (e.g., blue) and warm areas were shown with warmer colors (e.g., red and orange). Heat abnormalities in the microwave region, especially, denote regions of cancer. After processing the images of the data were sent to a color printer.

In the study, accepting histopathologic diagnosis as the gold standard, the validity (sensitivity, specificity, positive predictive value, negative predictive value) and consistenсу $\kappa$ values for mammography, US and RTM methods were measured.

We compared RTM results with X-ray mammography and the diagnostic value of RTM was statistically re- searched. Under this heading the sensitivity, specificity, positive-negative predictive value and consistency of RTM and US were evaluated compared to mammography.

Again we compared RTM results with X-ray mammography, the diagnostic value of RTM for size of mass and presence of microcalcification compared to mammography was researched, and the success of RTM in identifying lesions in different breast densities was evaluated.

\section{Statistical analysis}

The statistical analysis descriptively presents the number and percentage distributions, mean \pm standard deviation, median, minimum and maximum values. Validity analysis was examined as sensitivity, specificity, positive predictive value and negative predictive value compared to the gold standard of the scanning tests. Consistency in measurements was examined using the $\kappa$ value.

\section{Results}

The average age of patients was $48.5 \pm 10.3$ and median age was 48 (28-81). Evaluating the breast parenchyma structure of patients with mammography $22 \%(52 / 237)$ were BI-RADS I, 49\% (116/237) were BI-RADS II, 14\% (34/237) were BI-RADS III and 15\% (35/237) were BI-RADS IV.

Of patients with biopsy taken 33 (57.9\%) were malignant and 24 (42.1\%) were benign histopathologically. Mammography and US examination diagnosed 16 cases as BIRADS IV A, 21 as BI-RADS IV B, 4 as BI-RADS IV C and 16 as BI-RADS V.

When histopathology is accepted as the gold standard among diagnostic methods, the sensitivity, specificity, and positive-negative predictive value for RTM were $90.9 \%$, $20.8 \%, 61.2 \%$ and $62.5 \%$, respectively. Consistency was very low at 0.129 . The same values for mammography were 93.9\%, 43.3\%, 57.4\% and 33.3\%, respectively. Consistency was evaluated as good at 0.622 . Ultrasound examination values were $84.8 \%, 56.7 \%, 58.3 \%$ and $44.4 \%$, respectively. Consistency was good at 0.717 . For mammography and US in situations where a malignant mass was observed sensitivity, specificity, positive and negative predictive values were $96.7 \%, 60.8 \%, 47.7 \%$ and $65.8 \%$, respectively, with consistency very good at 0.915 (Table 1).

Accepting pathology as the gold standard an ROC curve was drawn for RTM and mammography methods. For RTM the area under the curve was $53.3 \%$ while mammography had $86.1 \%$ area under the curve. In other words, while a patient with breast cancer has an $86.1 \%$ possibility of having a suspicious positive on mammography, with RTM this rate is $53.3 \%$ (Fig. 2 ).

When we compared RTM results with X-ray mammography results, sensitivity, specificity, and positive-negative predictive values for RTM examination are $87 \%, 81.4 \%$, $58 \%$ and $95.5 \%$. Consistency is good at 0.582 . For US examination the same values are $85.2 \%, 98.9 \%, 95.8 \%$ and $95.8 \%$, respectively. Consistency is very high at 0.875 (Table 2).

When we compared RTM results with X-ray mammography results for microcalcification diagnosis, the sensitivity, specificity, and positive-negative predictive values for 

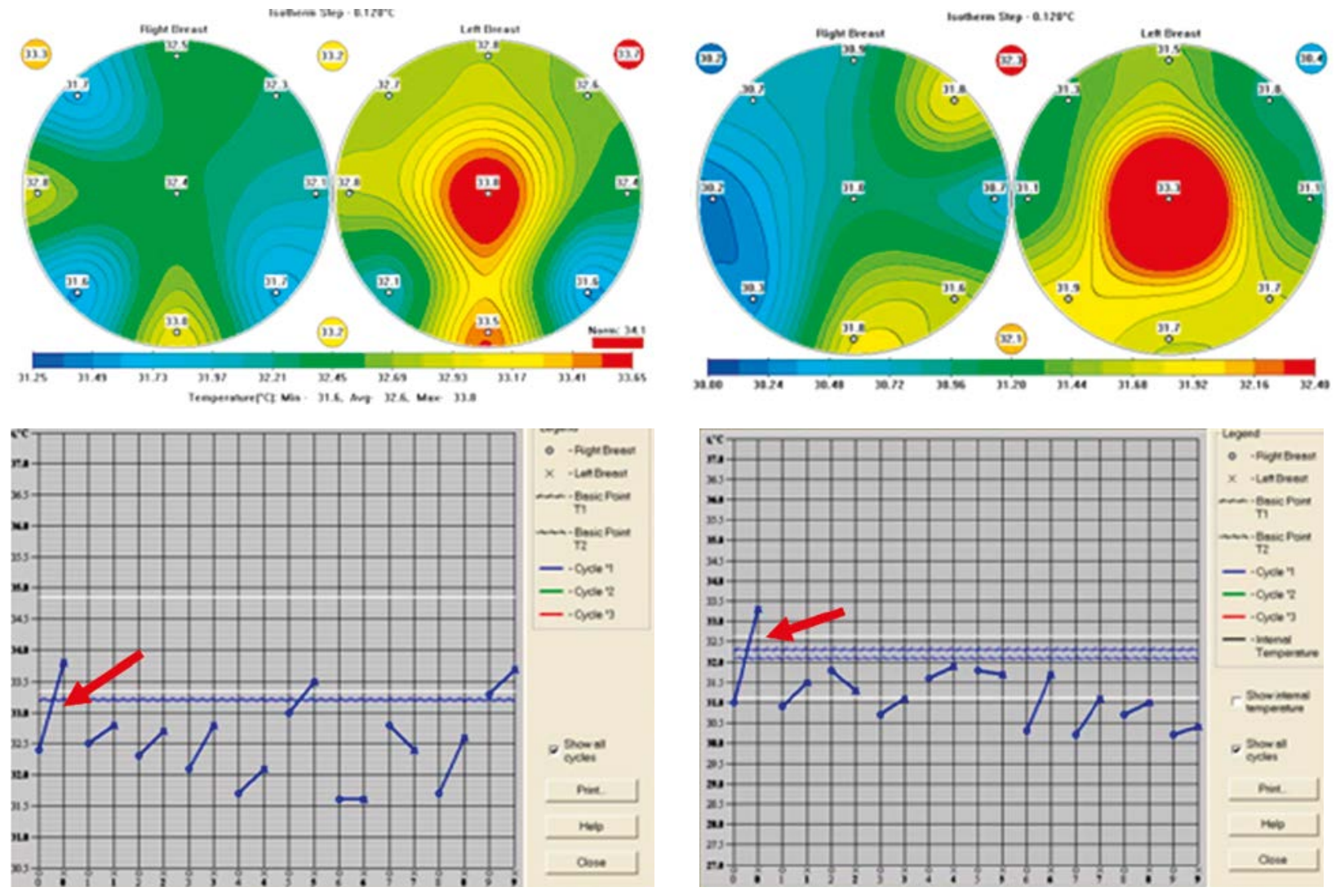

RTM report: Increase of the temperature in the tissue and skin on the central part of the left breast. The temperature was measured as $34.5^{\circ} \mathrm{C}$ (red arrow).
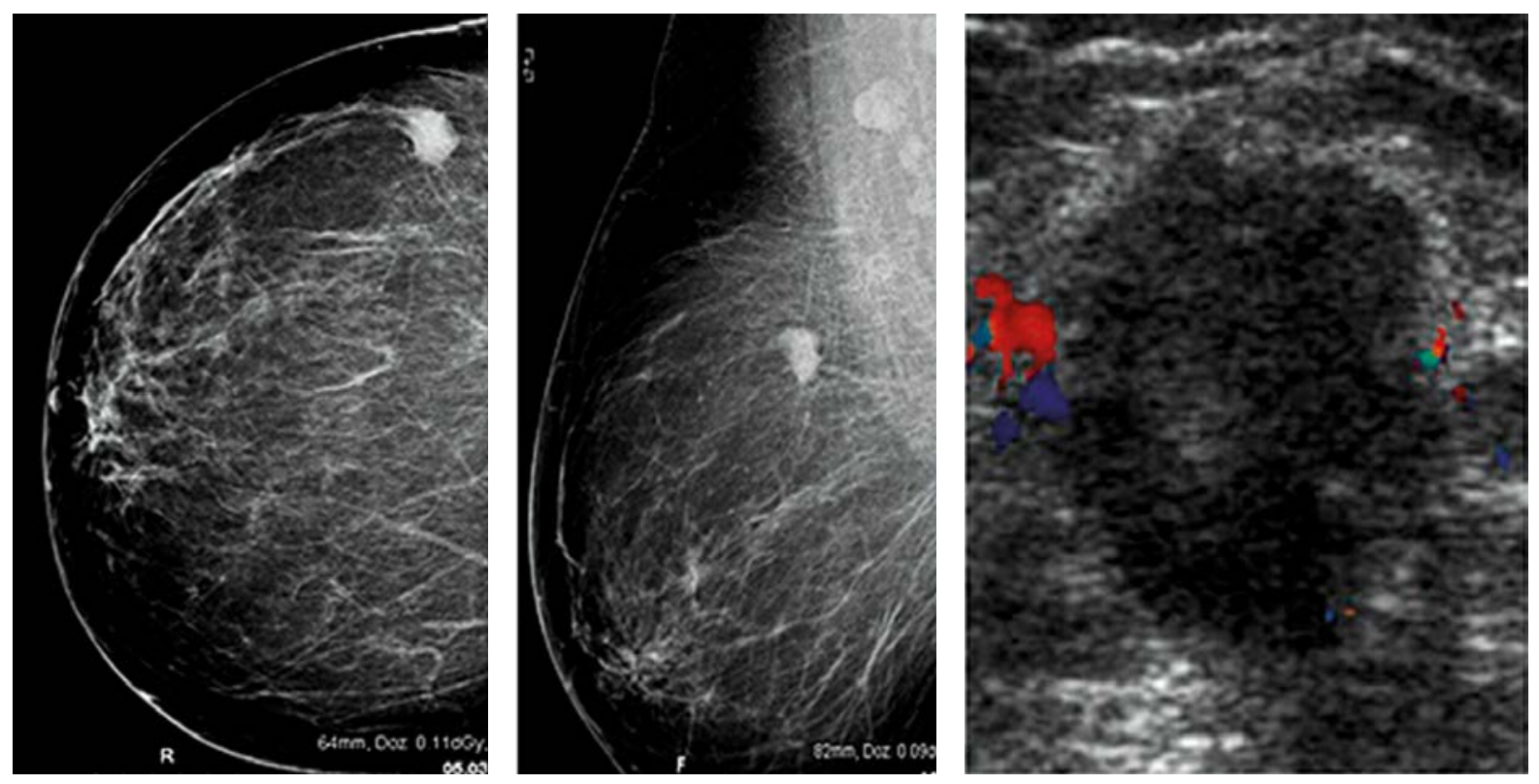

Mammography and USG: In the left breast upper outer region, slightly irregular contours, approximate dimensions of $18 \times 14 \mathrm{~mm}$ hypoechoic mass, in view of BI-RADS 4C lesion

Histopathological diagnosis: Invasive ductal carcinoma and invasive cribriform carcinoma in left breast

Fig. 1. A case: RTM positive 56-year-old patient

RTM were $86.7 \%, 80 \%, 92.9 \%$ and $66.7 \%$. Consistency was at a good level of 0.625. The same values for RTM examination in the absence of microcalcification were recorded as $87.2 \%, 18.5 \%, 50.7 \%$ and $49.3 \%$, respectively. Consisten- cy was good at 0.536 . That is, in patients with microcalcification the specificity, positive and negative predictive values for the RTM method were higher than for patients without microcalcification. Consistency in both cases was 
Table 1. Diagnostic value of imaging methods according to histopathology results $(n=57)$

\begin{tabular}{|c|c|c|c|c|c|}
\hline Diagnostic method & Sensitivity (\%) & Specificity (\%) & $\begin{array}{c}\text { Positive predictive } \\
\text { value (\%) }\end{array}$ & $\begin{array}{c}\text { Negative predictive } \\
\text { value (\%) }\end{array}$ & $\kappa$ value \\
\hline RTM & 90.9 & 20.8 & 61.2 & 62.5 & 0.129 \\
\hline Mammography & 93.9 & 43.3 & 57.4 & 33.3 & 0.622 \\
\hline US & 84.8 & 56.7 & 58.3 & 44.4 & 0.717 \\
\hline Mammography ve US & 96.7 & 60.8 & 47.7 & 65.8 & 0.915 \\
\hline
\end{tabular}

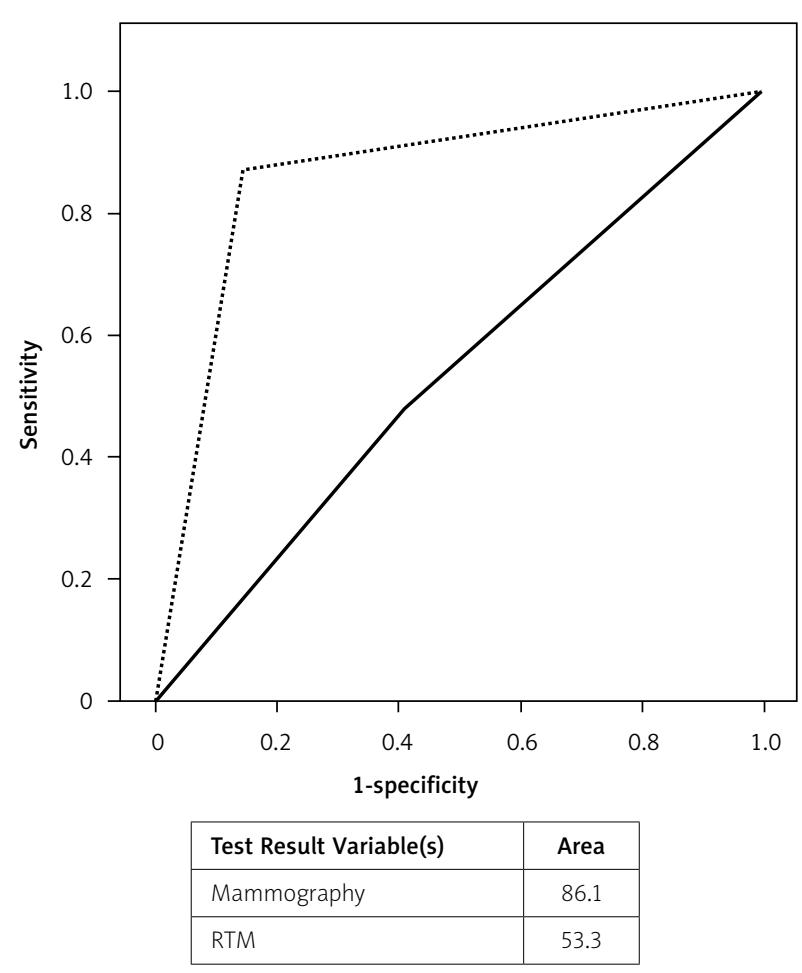

Source of the curve

....... Mammography - RTM

Fig. 2. ROC curves for RTM and mammography accepting pathology as the gold standard

at moderate levels, though higher for patients with microcalcification than for those without (Table 3).

The sensitivity, specificity, and positive-negative predictive values of RTM for determining lesions in different breast structure are as follows: BI-RADS I breast structure sensitivity $75 \%$, specificity $77.8 \%$, positive predictive value $60 \%$, negative predictive value $87.5 \%$ and consistency good at 0.494. BI-RADS II breast structure sensitivity was $93.1 \%$, specificity was $86.2 \%$, positive predictive value was $69.2 \%$, negative predictive value was $97.4 \%$ and consistency was at a good level of 0.711. BI-RADS III breast structure sensitivity was $100 \%$, specificity $100 \%$, positive predictive value $25 \%$, negative predictive value $94.1 \%$ and consistency low at 0.338 . BI-RADS IV breast structure sensitivity was $85.7 \%$, specificity $71.4 \%$, positive predictive value $42.9 \%$, negative predictive value $42.9 \%$ and consistency low at 0.416 (Table 4).

The consistency of RTM for lesion diagnosis in BI-RADS II breast structure is higher than the consistency of mammography. Mammography of dense breast tissue such as BI-RADS III and BI-RADS IV is at moderate to low levels.

The sensitivity, specificity, and positive-negative predictive values for US examination of different breast structures are as follows: BI-RADS I sensitivity $87.5 \%$, specificity $100 \%$, positive predictive value $100 \%$, negative predictive value $94.7 \%$. Consistency was very high at 0.906 . BI-RADS II: sensitivity $89.7 \%$, specificity $97.7 \%$, positive predictive value $92.9 \%$, negative predictive value $96.6 \%$ and consistency very good at 0.884 . BI-RADS III: sensitivity $50 \%$, specificity $100 \%$, positive predictive value $100 \%$, negative predictive value $97 \%$ and consistency good at 0.653 . BIRADS IV: sensitivity $71.4 \%$, specificity $100 \%$, positive predictive value $100 \%$, negative predictive value $93.3 \%$ and consistency very good at 0.800 (Table 5).

\section{Discussion}

This study is the first study in the literature that evaluates RTM in relation to mass size. In our study we examined the sensitivity, specificity and positive-negative values for RTM according to mass size. The specificity and positive predictive value of RTM for masses $2 \mathrm{~cm}$ and above were higher than for those below $2 \mathrm{~cm}$. For masses below $2 \mathrm{~cm}$ in size the negative predictive value of RTM was higher when compared with mammography.

The aim of breast imaging methods is to identify breast cancer in the early stages and to distinguish from different types of breast disease. In choosing a scanning method the most frequently used methods chosen by both clinicians and radiologists are mammography, ultrasonography and magnetic resonance imaging $[2,3,8]$.

The thermal imaging system breast radiothermometry examination (RTM) measures and analyses the tempera-

Table 2. Sensitivity, specificity, and positive-negative predictive values of diagnostic methods according to mammography results $(n=237)$

\begin{tabular}{|lcccc} 
Diagnostic method & Sensitivity (\%) & Specificity (\%) & $\begin{array}{c}\text { Positive predictive } \\
\text { value (\%) }\end{array}$ & $\begin{array}{c}\text { Negative predictive } \\
\text { value (\%) }\end{array}$ \\
RTM & 87.0 & 81.4 & 58.0 & 95.5 \\
US & 85.2 & 98.9 & 95.8 & 9.582 \\
\end{tabular}


Table 3. Sensitivity, specificity, and positive-negative predictive values of RTM according to mass size accepting mammography as gold standard

\begin{tabular}{|c|c|c|c|c|c|}
\hline Diagnostic method & $\begin{array}{l}\text { Sensitivity } \\
\text { (\%) }\end{array}$ & Specificity (\%) & $\begin{array}{c}\text { Positive } \\
\text { predictive value } \\
\text { (\%) }\end{array}$ & $\begin{array}{c}\text { Negative } \\
\text { predictive value } \\
\text { (\%) }\end{array}$ & $\kappa$ value \\
\hline $\begin{array}{l}\text { RTM } \\
(\text { mass size } \geq 2 \mathrm{~cm})^{*} \\
(n=45)\end{array}$ & 88.9 & 83.3 & 88.9 & 83.3 & 0.722 \\
\hline $\begin{array}{l}\text { RTM } \\
\text { (mass size }<2 \mathrm{~cm}) \\
(n=123)\end{array}$ & 89.5 & 76.9 & 41.5 & 97.6 & 0.451 \\
\hline $\begin{array}{l}\text { RTM (microcalcification - yes) } \\
(n=20)\end{array}$ & 86.7 & 80 & 92.9 & 66.7 & 0.625 \\
\hline $\begin{array}{l}\text { RTM (microcalcification }- \text { no }) \\
(n=217)\end{array}$ & 87.2 & 18.5 & 50.7 & 49.3 & 0.536 \\
\hline
\end{tabular}

*In cases with more than one mass, the size of the largest mass was noted

Table 4. Sensitivity, specificity, and positive-negative predictive values of RTM for identifying lesions in different breast structures $(n=237)$

\begin{tabular}{|lccccc|}
\hline $\begin{array}{l}\text { Diagnostic method } \\
\text { RTM }\end{array}$ & Sensitivity (\%) & Specificity (\%) & $\begin{array}{c}\text { Positive predictive } \\
\text { value (\%) }\end{array}$ & $\begin{array}{c}\text { Negative predictive } \\
\text { value (\%) }\end{array}$ & к value \\
\hline BI-RADS I $(n=52)$ & 75 & 77.8 & 60 & 87.5 & 0.494 \\
\hline BI-RADS II $(n=116)$ & 93.1 & 86.2 & 69.2 & 97.4 & 0.711 \\
\hline BI-RADS III $(n=34)$ & 100 & 100 & 25 & 94.1 & 0.338 \\
\hline BI-RADS IV $(n=35)$ & 85.7 & 71.4 & 42.9 & 95.2 & 0.416 \\
\hline
\end{tabular}

Table 5. Sensitivity, specificity, and positive-negative predictive values of US examination to identify lesions in different breast structures $(n=237)$

\begin{tabular}{|lccccc|}
\hline $\begin{array}{l}\text { Diagnostic method } \\
\text { US }\end{array}$ & Sensitivity (\%) & Specificity (\%) & $\begin{array}{c}\text { Positive predictive } \\
\text { value (\%) }\end{array}$ & $\begin{array}{c}\text { Negative predictive } \\
\text { value (\%) }\end{array}$ & $\kappa$ value \\
\hline BI-RADS I & 87.5 & 100 & 100 & 94.7 & 0.906 \\
\hline BI-RADS II & 89.7 & 97.7 & 92.9 & 96.6 & 0.884 \\
\hline BI-RADS III & 50.0 & 100 & 100 & 97.0 & 0.653 \\
\hline BI-RADS IV & 71.4 & 100 & 100 & 93.3 & 0.800 \\
\hline
\end{tabular}

ture of the breast surface (infrared thermometry) and the internal tissue (microwave thermometry) and is a method to aid in the diagnosis of breast cancer. The RTM device measures electromagnetic waves transmitted through breast tissue to identify the diseased area. Microwave thermometry is a technology developed in recent years which aims to diagnose cancer using thermal activity of cancer cells in breast tissue, before structural changes occur. It is reported that this method has the potential to differentiate between fibrocystic cell changes and cancer cell changes. Examination does not involve radiation. All areas, including the axilla, can be imaged [3-10].

Microwave radiometry was used as a heat method for breast examination for the first time by Barett et al. In a study of 5000 patients the method was to expose tissue to heat at microwave frequencies. Sensitivity and specificity were $70 \%$. While microwave thermography affects all breast tissue, infrared thermometry only affects the epidermis, making microwave thermography more produc- tive. Combined use of RTM and infrared thermometry has a sensitivity of $90 \%$ while specificity is $50 \%[5,9,10]$.

While it may not be used widely in medical applications, the first study to use microwave radiometry as a diagnostic method for breast cancer was published in 1977 [11]. Many years later, developed from trustworthy and simple studies, a computer-based, always ready microwave radiometry device that does not require calibration was developed (RTM-01-RES) [11-13]. This device was the RTM device used in our study. It records the temperature increase caused by masses $3-5 \mathrm{~cm}$ deep in the breast tissue. In malignant masses a fast temperature increase is directly correlated with the speed of growth of the mass. On thermograms of breast cancer this is identified as hyperthermic areas, due to their high metabolic process and good vascularization. This method, in a study of 1000 patients, had sensitivity of $85-94 \%$ and specificity of $75-80 \%$. Both methods, mammography and RTM, when used together can reduce false negative results by $1-3 \%[14-16]$. 
The radiothermometry examination can be used not only for breast cancer, but also for diagnosis of urinary [17], anterior eye [18], and thyroid [19] diseases, and rheumatoid arthritis [20].

Burdina et al. [21] in a study of 1000 patients found that RTM results correlated with pathology; the sensitivity of this method was $85-94 \%$, specificity was $75-80 \%$ and the RTM results overlapped with those of mammography. Avramenko et al. [15] studied non-palpable masses in 200 female patients using RTM scanning together with US and mammography. They concluded that the sensitivity of the three methods was $86.7 \%, 75.8 \%$ and $88.3 \%$ with specificity of $75.2 \%, 80.0 \%$ and $83 \%$.

In this study, as in other studies, suspicious cases not only on RTM, but also on mammography and US, were sent for histopathologic diagnosis. As a result, positive results were expected to be higher. In other words, sensitivity and positive predictive values in this study were higher than specificity and negative predictive values. Similar results are seen in Table 5 where the specificity and negative predictive values between mammography and histopathology are lower than sensitivity and positive predictive values. This situation results from a higher proportion of malignant cases being directed for histopathologic diagnosis.

When we compared RTM results with X-ray mammography results, the validity results for RTM (sensitivity, specificity, positive and negative predictive values) are $87.0 \%$, $81.4 \%, 58 \%$ and $95.5 \%$ respectively, with the consistency value at a good level of $\kappa=0.582$. This result indicates that there is a high level of compliance between both diagnostic methods.

In other studies the high specificity value may be due to histopathologic diagnosis for suspicious cases based on clinical results and/or on RTM. In our study the criterion for the invasive method of biopsy was suspicion on mammography and US. As a result the moderate values for specificity and negative predictive values compared to other studies are natural [13-16, 21]

There are no studies in the literature that evaluate RTM in relation to mass size. In our study we examined the sensitivity, specificity and positive-negative values for RTM according to mass size. RTM examination had sensitivity, specificity and positive-negative values for masses $2 \mathrm{~cm}$ and above of $88.9 \%, 83.3 \%, 88.9 \%$ and $83.3 \%$. For masses below $2 \mathrm{~cm}$ in size the sensitivity, specificity and positive-negative values for RTM were $89.5 \%, 76.9 \%, 41.5 \%$ and $97.6 \%$. In conclusion, the specificity and positive predictive value of RTM for masses $2 \mathrm{~cm}$ and above were higher than for those below $2 \mathrm{~cm}$. For masses below $2 \mathrm{~cm}$ in size the negative predictive value of RTM was higher. Sensitivity in both cases was similar. There is no study in the literature evaluating according to mass size.

Sensitivity, specificity and positive-negative values of RTM examination in the presence of microcalcification were $86.7 \%, 80.0 \%, 92.9 \%$ and $66.7 \%$, respectively. In the absence of microcalcification the sensitivity, specificity and positive-negative values were $87.2 \%, 18.5 \%, 50.7 \%$ and $49.3 \%$, respectively. The conclusion is that in cases with microcalcification the specificity, positive and negative predictive values were higher than in cases without microcalcification. The reason for this may be the possibility that the lesion is large or that it may be late stage. However, there is no study in the literature that evaluates based on the presence of microcalcification.

Additionally, the sensitivity, specificity and positive-negative values of RTM in different breast structures were researched. The results for BI-RADS I breast density were sensitivity $75 \%$, specificity $77.8 \%$, positive predictive value $60 \%$ and negative predictive value $87.5 \%$. For BI-RADS II breast density sensitivity was $93.1 \%$, specificity $86.2 \%$, positive predictive value $69.2 \%$ and negative predictive value $97.4 \%$. BI-RADS III sensitivity was $100 \%$, specificity was $100 \%$, positive predictive value was $25 \%$ and negative predictive value was $94.1 \%$. For BI-RADS IV breast density sensitivity was $85.7 \%$, specificity $71.4 \%$, positive predictive value $42.9 \%$ and negative predictive value $42.9 \%$. The consistency of RTM for lesion diagnosis in BI-RADS II breast density was higher than for mammography. On mammography for dense breast tissue types such as BI-RADS III and BI-RADS IV values were moderate and low. The sensitivity, specificity, and positive-negative predictive values of US for lesion detection in different breast densities were higher than for RTM. There is no study in the literature with information on this topic.

Our study comprised 237 patients. Of these, only 57 were sent for histopathologic diagnosis of masses found on mammography and US. The low case number and low number of patients with histopathologic diagnosis by biopsy are important limitations. However, the research into sensitivity for lesion recognition based on breast density, presence of microcalcification and mass size is an advantage not found in the literature.

In conclusion, identification of lesions in the breast and presence of microcalcification by RTM shows that it is more trustworthy compared to mammography. Accepting mammography as the gold standard, the validity results for RTM show a good level of conformity between the two methods. When evaluated based on the area below the ROC cure and compared to mammography, RTM is sufficiently successful at evaluating positive and negative cases. These results show that RTM is not appropriate as a single scanning method. If this method is used, it is more appropriate to use it with basic scanning methods such as mammography and US. As the experience in this area is limited, we believe that studies with higher case numbers will further determine the diagnostic contribution of RTM.

The authors declare no conflict of interests.

\section{References}

1. Aberle DR, Chiles C, Gatsonis C, Hillman BJ, Johnson CD, McClennan BL, Mitchell DG, Pisano ED, Schnall MD, Sorensen AG; American College of Radiology Imaging Network. Imaging and cancer: research strategy of the American College of Radiology Imaging Network. Radiology 2005; 235: 741-51.

2. Niedźwiecki S, Piekarski J, Jeziorski A. Influence of the imaging method and histopathological features on preoperative size prediction of ductal carcinoma in situ (DCIS) of the breast. Prz Menopauzalny 2013; 17: 459-63. 
3. Popiela TJ, Kibil W, Herman-Sucharska I, Urbanik A. The use of magnetic resonance mammography in women at increased risk for developing breast cancer. Wideochir Inne Tech Malo Inwazyjne 2013; 8: 55-62.

4. Leung JW. Screening mamograhpy reduced morbidity of breast cancer treatment. AJR Am J Roentgenol 2005; 184: 1508-9.

5. Bardati F, ludicello $S$. Modeling the visibility of breast malignancy by a microwave radiometer. IEEE Trans Biomed Eng 2008; 55: 214-21.

6. Fear EC. Microwave imaging of the breast. Technol Cancer Res Treat 2005; 4: 69-82.

7. Burdina LM, Vaisblat DS, Vesnin MA, Konkin AV, Lyashekov NG, Naumkina, NN et al. Detection of breast cancer with microwave radiometry. Mammology 1998; 3-12.

8. Berman CG. Recent advances in breast-specific imaging. Cancer Control 2007; 14: 338-49.

9. Burdina LM, Pinkhosevich IG, Khailenko VA, Vesnin SG, Tikhomirova NN. Experience of Using RTM-01-RES Radiometer for Breast Cancer Diagnosis Radiology' 2002. Proceedings of the III Russian Scientific Forum "X-Ray Diagnostics and X-Ray Therapy in Clinic of XXI", Moscow, Aviaizdat 2002; 49-50.

10. Gledo I, Pranjic N, Drljević K, Prasko S, Drljevic I, Brzeziński P. Female breast cancer in relation to exposure to medical iatrogenic diagnostic radiation during life. Contemp Oncol (Pozn) 2012; 16: 551-6.

11. Barrett AH, Myers PC, Sadowsky NL. Detection of breast cancer by microwave radiometry. Radio Sci 1977; 12: 167.

12. Mashal A, Booske JH, Hagness SC. Toward contrast-enhanced microwave-induced thermoacoustic imaging of breast cancer: an experimental study of the effects of microbubbles on simple thermoacoustic targets. Phys Med Biol 2009; 54: 641-50.

13. Fogiel M, Zbroch T, Knapp P, Knapp P. Microwave thermography in the assessment of breast pathology. Med Wieku Rozwoj 2002; 6 ; 63-73. [Article in Polish].

14. Sdvigkov AM, Vesnin SG, Kartasheva AF, Bjahov MU, Gurtvoy IJ, Borisov VI, et al. Role of Microwave Radiometry in Mammology. Actual Problems of Mammology 2000; 28-40.

15. Avramenko GV. Use of radiothermometry in the screening of nonpalpable breast neoplasms Vestn Rentgenol Radiol 2007; 9-12.

16. Barrett AH, Myers PhC, Sadovsky NL. Microwave thermography in the detection of breast cancer. AJR Am J Roentgenol 1980; 134: 365-8.

17. Avdoshin VP, Andriukhin MI, Ol'shanskaia EV. Parenteral litholytic therapy of urate urolithiasis. Urologiia 2008; (4): 9-10, 12-3.

18. Orlov PI. Infrared radiothermometry in the differential diagnosis of dystrophic and inflammatory diseases of the anterior eye. Vestn Oftalmol 2008; 124: 19-22. [Article in Russian].

19. Vetshev PS, Chilingaridi KE, Zolkin AV, Vesnin SG, Gabaidze DI, Banny̆ DA. Radiothermometry in diagnosis of thyroid diseases. Khirurgiia (Mosk) 2006; (6): 54-8. [Article in Russian].

20. Bazhanov NN, Semenova EV, Ginzburg LI. The use of radiothermometry in patients with rheumatoid arthritis. Ter Arkh 1992; 64: 93-6.

21. Burdina LM, Vaisblat AV, Vesnin SG, Tihomirova NN. Possibility to diagnose breast cancer by measuring natural electromagnetic radiation from tissues (radiothermometry). Mammology 1997; $17-22$.

\section{Address for correspondence}

\section{Fatma Uysal}

Çanakkale Onsekiz Mart University

17100 Çanakkale, Turkey

e-mail: afatmauysal@gmail.com

Submitted: 28.10 .2013

Accepted: 25.02 .2014 